\title{
Etymologisches aus den permischen Sprachen
}

Syrj. veža 'heilig'.

Dieses Wort hat Uotila FUF XXVI 186-9 eingehend behandelt und ausführliche Belege für Form und Bedeutung des syrj. Wortes aus den Quellen zusammengestellt, weshalb wir hier das Vorführen der Belege unterlassen und diesbezüglich bloss auf UotiLas erwähnten Artikel verweisen.

UотіLA kommt a.a.O. zum folgenden Resultat:

1. Das bereits in den syrj. Sprachdenkmälern belegte Wort veza 'heilig', das heute besonders in Ausdrücken, wie vež-ai 'Taufvater', vež-ań 'Taufmutter' (<*veža-ai, *veža-añ), vežanil 'Tauftochter', veža-pi 'Taufsohn', ferner veža-lun 'Sonntag; (auch:) Woche', veza-pera 'Zeit von Weihnachten bis zum heiligen Dreikönigsfeste (святки)', veža-va 'das geweihte Wasser', (Wied.) veža-dyr 'Feiertagszeit' gebräuchlich ist, stellt offensichtlich eine Ableitung mittels des Adjektivsuffixes -a dar.

2. Da veža im grössten Teil der obigen Fügungen dieselbe Bedentung hat, wie russ. крестный 'sich auf die heilige Taufe beziehend, Tauf-; zum Kreuze gehörig, Kreuz-' (Pawl.) (z.B. к. отецъ 'Taufvater', к-ая мать 'Taufmutter', к. сынъ 'Taufsohn'; vgl. крестъ 'Kreuz'), ist es möglich, dass syrj. veža ein Derivat von syrj. (Wied.) vež 'Kreuz, Quere', (Wichm.) tuj-ve $Z$ 'Wegkreuz, Wegscheide' ist (zu diesen vgl. russ. крестъ на крестъ 'kreuzweise, ins Kreuz', крест ́' '. . . . Kreuz, Scheideweg', перекрестокъ 'Kreuzweg'), „d.h. dass veža eine sog. Übersetzungsentlehnung ist». Hierfür spreche auch syrj. I pernai 'Taufvater', perńan 'Taufmutter' $\left(<^{*}\right.$ perna-ai, * ${ }^{\text {perna- }}$ ań) von perna 'Halskreuz (bei den Griechisch-Katholischen)'.

3. "Die obige Erklärung von syrj. veža ist jedoch keineswegs als sicher zu betrachten. Es ist möglich, dass dieses Wort schon zu der Terminologie der alten syrjänischen Religion gehörte. Zu beachten, dass veža 'heilig' schon bei Stephan, dem Apostel der Syrjänen (in der zweiten Hälfte des 14. Jh.), vorkommt." 
Bevor wir weitergehen, müssen wir noch darauf hinweisen, dass - wie auch Uotila bemerkt - das Wort für 'Kreuz' in PO (bei GeneTz) riz lautet (davon tyj-viz 'Kreuzweg'); auch V. I. LyTKIN hat aus PO (Jazv.) tui-viz (Vok. 83), bzw. tyiriz (Drperm. 125) 'перекресток дороги'. Bei LYTkIN finden wir ausserdem noch zwei Belege für dieses Wort aus PO: vizai 'крестный отец' (Vok. 83) und pad-vižөn 'крест-на-крест, пересекая одно другим' (Drperm. 140). Über diese Lautentsprechung, die auf ursprüngliches * $e$ hinweist, s. V. I. LYTKIN (Vok. 82 ff., Drperm. 90-91), E. ITKonex (FUF XXXI 310-11), Toivonex (FUF XXXII 131, 132).

V. I. Lytkis führt (Vok. 83, Drperm. 125) neben der Uotilaschen Deutung (in diesem Falle sei für veža als ursprüngliche Bedeutung 'крестный, крестом освященный' anzusetzen) noch eine andere Erklärung an, die auf G.S. LYTkıN zurückgeht. Letzterer verbindet nämlich in seinem Wörterbuch veža mit syrj. vež 'зеленый, желтый, светлый ...' [(Wichm.-Uot.) V S L U P $v e z$, I $\imath \grave{e} z$ 'grün' (V U I), 'hellgrün(z.B. Saat), grasgrün' (S L),' gelb' (P); (Gen.) PO viz 'gelb, grün', (LYTk. Vok. 82, Drperm. 125) РО (Jazv) viz 'желтый, зелёный'] ( wotj. voz 'grün, unentwickelt, klein'; s. auch E. ITkoneN FUF XXXI 311), und V. I. LytKin nimmt als Grundbedeutung 'свет.тый, блеклый' an (wie in V vez-jecdžid 'бело-бело' usw.).

Wir kommen noch w.u. auf diese Erklärung zurück. Vorläufig müssen wir feststellen, dass uns diese letztere Deutung in dieser Form kaum befriedigen kann. Ich selbst habe ja auch in $\mathrm{V}$ als Bedeutungen von vez nicht nur 'grün', sondern auch 'hell' aufgezeichnet (und V vez gi bedeutet 'schäumende Welle, Welle mit (hellen, weissen) Wogenkämmen'), V vežedni bedeutet nicht nur 'grün machen', sondern auch 'hell machen', dennoch ist eine Bedeutungsentwicklung 'hell, fahl' (NB. nicht 'glänzend' od. 'leuchtend') $\rightarrow$ 'heilig' nicht eben etwas Selbstverständliches (zwar bedeutet vez in $\mathrm{P}$ nach Wiedemans 'hell, glänzend', vež tšsotšköm 'glänzend weiss'), und wir bekommen keine Antwort auf die Frage, weshalb ein Adjektiv ('grün') noch ein Adjektivsuffix annehmen musste. [vez bedeutet zwar auch 'зелень; лугъ' (Lytk.), 'Grünes, Wiese' (Wied.), doch ist dies gewiss die sekundäre Bedeutung; weder WiEdemans, noch ich haben ein solches Substantiv aufgezeichnet ${ }^{1}$.] S. nocb w. unten.

Auch Uotilas Deutungsversuch ist nicht völlig überzeugend. ce ža lässt sich ja in gewissen Wendungen als Lehnüber-

1 Und auch wenn es 'das Grüne' bedeuten könnte (vgl. vins Grüne gehen"), hätte ja ve ̌̃ $a$ wiederum nur die Bedeutung 'grün'. 
setzung auffassen, und auch $v e^{z}$ lässt sich in Anwendungen, wie tui-rez 'Wegkreuz, Wegscheide', V pad-vez 'крест-накрест; kreuzweise' (z.B. V kutasni pad-vez genitni 'sie beginnen gegeneinander zu galoppieren'; pad-ıeža 'kreuzweise liegend (Adj.)'), sowie in Ableitungen (wie SL vežen munni 'vorbeigehen (ohne zu treffen), sich verfehlen', I U vezin' 'krenzweise, übers Kreuz ...' usw.) auf Grund von (Wied.) vež 'Kreuz' erklären, doch dies alles lässt sich nicht damit vereinbaren, dass (selbständiges) veža bereits seit sehr alten Zeiten und sozusagen auf dem ganzen syr.j. Sprachgebiet ausschliesslich 'heilig' bedeutet.

Wir haben es wohl in rezz 'Kreuz' und ręza 'heilig' mit zwei verschiedenen Wörtern zil tun.

Für veža liegt ja ein Deutungsversuch vor, der gewiss volle Beachtung verdient. (Bezüglich des Wortes vežz 'Kreuz' s.w.u.) WotjChrest. 131-2 schreibt Wichuaxis folgendes: "Gr rozo 'ein böser Geist, verursacht u.a. Krankheiten; treibt während der Winter- und Sommersolstitien (vozo-dì $r=$ 'die vozo-Zeit') herum, da man ihn (mit Arbeit, Geräusch u.a.) nicht stören darf. Vgl. syrjI. veza pera 'Zeit von Weihnachten bis zum heiligen Dreikönigsfeste (святки)'. - vožo-mad' 'Rätsel. (Mit Rätseln unterhält man sich vorzugsweise zur cožo-Zeit.)" Bei Munkácsi (VotjSz. 679, 53-4) finden wir folgende Angaben: SM J vožo:in-rožo 'Sternschnuppe, Meteor; Irrlicht; wilde Nelke'. S M G vozo-dir 'Zeit des In-vožo-Dienstes in den heissen Sommertagen' (S M): 'wotjakische Benennung der kirchlichen zwölf Tage von Weihnachten bis zum Fest der heil. drei Könige' (G). S in = vožo-dir, in-rozo 'Zeit der Sommerund Winter-Sonnenwende. ungefähr rom 20. Juni bis 20. Juli und vom 25. Dezember bis zum 6. Januar. In den Mittagsstunden dieser Tage ist jedwede Arbeit verboten, besonders Grasreissen, Graben, Waschen, Baden im Flusse. Ebenso ist es eine Entweihung dieser geheiligten Zeit, wenn man singt oder Lärm macht, schwarze Cregenstände (z.B. Kessel, Eisentiegel) ans Sonnenlicht bringt, sowie wenn man brennende Kohle zertritt, welche Sünden als Strafe Gewitter nach sich ziehen'. vož-mad' 'Rätsel, mit welchen man sich zur Zeit des vožo-dir unterhält'. S vožo-miž 'In-vožo's Forderung in bezug auf ein Opfer, das von jmdem rersprochen, aber noch nicht dargebracht wurde'. Das Udm.-russk. Slov. von 1948 hat vožodir 'святки'. (Ausführlicher über vožo s. Musk. NyK XXI 234-5, MSFOu. CII 135, WasILJEW MSFOU. XVIII 141-3, Holmberg MSFOu. XXXII 89 ff., Permalaisten uskonto $\left.159-60,174-5,203 .^{1}\right)$

1 Wotj. vožo als Name eines Geistes beruht gewiss auf einer späten Personifikation. 
Es ist wohl ein naheliegender Gedanke, dass wotj. vožo [-dir ( syrj. veža[-dir]) eig. 'verbotene [Zeit], geweihte [Zeit], Tabu[-Zeit]' bedeutet; vgl. auch syrj. (Wichm.-Uot.) I jēe-vez̄o (< vežol)-lin 'Butterwoche, Fastnachtswoche'. Und wie leicht sich aus dieser Bedeutung der Sinn 'heilig' entwickeln kann, ist ja zur Genüge bekannt. Wir wollen nur auf ein ostjakisches und ein samojedisches Beispiel hinweisen. Im Ostjakischen bedeutet (Karj.-Toiv. 170) Trj. $i i^{\prime} m^{\circ}$ "wenn du etwas tust, was man nicht tun darf (z.B. dich nach dem Tod deiner Eltern freust, Gott zuwiderhandelst, am Feiertag arbeitest usw.), so ist es $i i^{\prime} m^{\prime} "$; Ni lem $^{\prime}$ 'was durch den Anstand oder durch religiöse Gebräuche verboten ist; also $\mathrm{S}$ ü nd e'; $\mathrm{Kaz}_{i \varepsilon^{\prime} m^{\prime}}$ 'Heiligkeit, Unberührbarkeit (einer heiligen Stätte); Verbotenes, nicht Erlaubtes (Subst.); Sünde'; 0 ie'm 'Sünde', - und

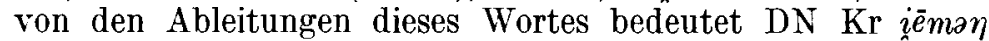

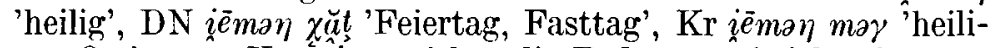
ger Ort' usw.; Kam ìemattä hat die Bedeutung '»sich schämen, sich genieren" (vor einem älteren Menschen), die Anstandsregeln befolgen, d.h. etwas nicht tun od. sagen, was beschämend ist ...' usw. (S. auch Paas.-Donn. 33-4.) Im Juraksamojedi-

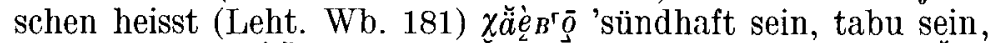

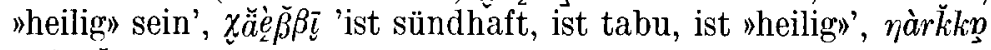

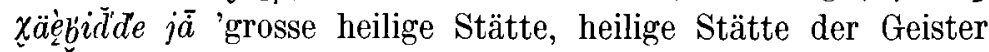

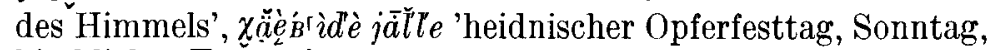
kirchlicher Festtag' usw.

Dass wir auf der richtigen Fährte sind, wenn wir die Herkunft von syrj. veža in dieser Richtung suchen, bezeugt ein Ausdruck, in dem vež als Grundwort von veža tatsächlich zur Bezeichnung einer magischen Handlung dient; ich habe in $\mathrm{V}$ (s. auch KSz. XII 248) folgende Wendung aufgezeichnet: vež-hiv uśkৎdni 'сглазить, пспортить, напустить порчу; beschreien, einen Schaden anzaubern' (eig. 'ein vež-Wort auf jem. werfen, fallen lassen') und Popov-Lytkin führt ebenfalls (p. 209) syrj. vežkil uśem im Sinne von порченье, порча an.

1 Sehr lehrreich sind noch KarJalainens diesbezügliche Bemerkungen (Die Religion der Jugra-Völker II 84): Die Wörter jem und pes - schreibt er hier - bedeuten "wahrscheinlich die Unberührbarkeit, Unverletzlichkeit eines Wesens oder Dinges...., also die "Heiligkeit" desselben gegenüber irgend welchen oder allen anderen Wesen und Dingen, folglich ungefähr das, was man in der religionswissenschaftlichen Literatur mit dem Worte $t a b u$ zu bezeichnen pflegt. Gegenwärtig versteht der Ostjake unter jem . . . alles das, was den religiösen Bräuchen und Schicklichkeitsbegriffen zuwiderläuft, also das 'nicht gestattete', und übersetzt diese Wörter [näml. jem und pəs], wenn er sie überhaupt noch kennt, ins Russische mit dem Worte grjeh 'Sünde', eine Bedeutung, die sich aus der vorhergehenden entwickelt hat: das die Unberührbarkeit, die Unverletzlichkeit Besitzende zu berühren, zu verletzen, ist jem, pas "Sünde».» Vgl. auch ebd. II 80. 
Die Bedeutung 'heilig', die sich - wie angenommen werden kann - ohne Hinzutun des Bischofs Stephan entwickelt hatte, kam dem Apostel der Syrjänen gewiss sehr gelegen, ebenso, wie er z.B. das Wort jen 'Gott' übernahm, das »in dieser Bedeutung offenbar schon in der alten Religion" der Syrjänen gebräuchlich war; ves entspricht dem wotj. in, in 'Himmel, Luft', fi. ilma 'Luft, Wetter' usw." (Uor. FUF XXVI 188-9).

Und wie allgemein das Wort in dieser Bedeutung war, dafür können wir noch auf einen wichtigen Beleg hinweisen. Auf wogulischem Gebiet gibt es wim Ural eine hohe Bergkette, deren Name auf Russisch Molebnyi Kameń, d.h. 'Gebets-Ural' und auf Wogulisch $i a l_{c} l_{(p \eta}$ ñor 'Heiliger Ural' heisst; auf dem Berg entspringt der rechte Nebenfluss der Lozva, der V i ž a i, auf Wogulisch $i \dot{a} l_{\text {( }} \eta i_{n} \vec{a}$ 'Heiliger Fluss'» (KanNisTo FUF XVIII 67). Den russ. Вижай $=$ wog. Jelpin $j \bar{a}$, einen Nebenfluss der Lozva erwähnt auch Munkácsi (VNGy. I 0243, II 016, 020, $0181,439,446,615,738)^{1}$; auch er führt die Benennung Верхвижайскій Камень od. Молебный Камень 'GebetsStein' [d.h. 'Gebets-Ural'] an (ebd. II 020). ${ }^{2}$ Er übersetzt die aus dem Syrjänischen übernornmene russische Benennung durch "szent bálvány öreg [folyója]", d.h. '[Fluss des] heiligen Götzen-Alten' und bemerkt, Вижай sei aus syrj. vižs, vi ̌̆ 'heilig' (in $v i c c^{\prime} k o$ 'heiliges Haus, Kirche') und $a j$ 'Alter' zusammengesetzt (op. c. II 016, 446, 738).

Der Name Вижай - dessen ersten Teil Munkácsi freilich nicht richtig erklärt - ist gewiss lehrreich und wichtig. Vor allem zeigt die Lautform, dass die syrj. Benennung viž-ai zweifellos aus dem Ostpermjakischen (Jazva-Permjakischen) ins Russische übernommen wurde (s. oben S. 165), und der gewiss schon in frühen Zeiten entlehnte Name hat - wie die wogulische Übersetzung zeigt - bereits zur Zeit der Übernahme nicht 'Taufvater', sondern 'heiliger Alter', 'heiliger' Greis' (vgl. Wied. ai 'Vater, Greis') bedeutet.

Wir dürfen wohl als Ergebnis unserer bisherigen Ausführungen feststellen, dass syrj. veža 'heilig' nicht mit syrj. vež 'Kreuz' verbunden werden kann und demnach keine Lehnübersetzung, sondern aller Wahrscheinlichkeit nach ein mit dem ebenfalls zu einem religiösen Termin gewordenen wotj. vožo identisches

1 Auch KarJalainen schreibt (RelJ. II 85): "Die Mündung des in die obere Lozva sich ergiessenden Flusses Vizhaj (wog. Jelpi $j$ jā 'Heiliger Fluss') ist so heilig, dass die Frauen, wenn sie sie befahren, im Boot nicht sitzen dürfen und dass auch die Männer ihr Haupt verhüllen und lautlos rudern."

2 Bemerkenswert ist, dass auch im Udora-Gebiet (in der Nähe von Cub) ein Fluss syrj. veż-aikka-ju und ebendort ein Berg syrj. vez-ainka-gera heisst. 
genuines syrj. Wort ist; beide Wörter wurzeln wohl mit ihrer Bedeutung im alten Glauben ihres Volkes.

Was mag nun die Etymologie dieses permischen Wortes sein? Sowohl syrj. veza, als auch wotj. vožo weisen offenbar das Adjektivsuffix syrj. $-a \sim$ wotj. $-o$ auf, sind also Ableitungen von einem Substantiv. ${ }^{1}$ Eben mit Rücksicht auf diesen Umstand haben wir ja oben bemerkt, dass Lytкiss Versuch, veza mit dem Adjektiv vež 'grün' zu verbinden, bzw. von diesem herzuleiten, in dieser Form nicht überzeugend wirken kann. Ganz anders werden wir aber die Sache beurteilen, wenn wir in Betracht ziehen, dass dasselbe wotj. Wort auch 'Zorn, Grimm' (Munk.), 'Zorn' (Wichm.) bedeutet (z.B. Munk. S vožez potäm 'er zürnte, ist böse od. grimmig geworden') und dasselbe syrj. ve $e z$ [in einigen Redensarten] die Bedeutung 'Begierde, Lust, Neid' hat, sogar 'Zorn' bedeuten kann, also jedenfalls als Substantiv gebräuchlich ist [z.B. Wichm. V vezz pet$k \varrho d n i, \mathrm{U}$ vez petke.dnis 'Begierde, Lust erregen' (V), 'böse machen, aufbringen, erzürnen' (U); $P$ veželitini 'eifersüchtig sein' usw.; (in meinen Aufzeichnungen z.B.) $\mathrm{V}$ vez petkedni 'Verlangen, Begierde, Neid erregen, hervorrufen, auslösen', $\mathrm{V}$ vežegtini, vežkti-, U vežektini, Le vežE.ktini 'eifersüchtig sein', (V auch:) 'nicht lieben, hassen' usw.J. Diese Bedeutungen gehen - wie Hakulisex MSFOu. XCVIII 189 ff. bezüglich dieser und der mit ihnen etymologisch zusammengehörenden Wörter anderer fiugr. Sprachen gezeigt hat $(\sim$ fi. viha 'Zorn, Hass, Feindschaft', rihanta 'grün, grünend, unreif', viheriä 'grün', lapp. N râšse 'hate, hatred, grudge', md. ožo 'gelb', tscher. $u z z a \cdot r$ usw. 'grün') ${ }^{2}$, auf die Grundbedeutung 'grün, etwas Grünes' ( $\rightarrow$ 'bitter') zurück. (Über indoeuropäische Beziehungen sowohl in Form als auch in Bedeutung vgl. SETÄLÄ FUF VIII 79 Fussnote, XIII 471, HaKulisen l.c. 194-5, Collinder FUV $140-1$.) Die erwähnte Zusammenstellung des syrj. und wotj. Wortes mit den zitierten fiugr. Wörtern ist ja einwandfrei; WichMaxx WotjChrest. 131 verbindet syrj. $\sim$ wotj. $\sim$ fi., Cotila SyrjChrest. 175 syrj. $\sim$ wotj. $\sim$ fi. $\sim$ md. tscher.; auch E. ITkoxex vertritt (FLF XXXI 182, 311)

1 Ganz verfehlt ist es freilich, wenn JEMEL'JA vov (GrVotj. 105) wotj. vožo auf folgende Weise erklären will: жо ̈о маскированный, соб̆. мифич. существо в период солноворота, витающее на земле (ср, voš-tменять). Unrichtig ist auch McNkAcsis Versuch (NyK XXI 235), vožo mit voż 'Ort, wo zwei Flüsse od. Wege zusammenfliessen; Flussmündung, Wegscheide' zu verbinden. - Interessant ist, dass bereits Pervuchin und Gavrilov - freilich mit einer unrichtigen Begründung - an einen Zusammenhang zwischen wo $\approx o$ und co ̇ 'grün' denken (s. Muxk. Nyh XXI 234).

${ }^{2} \mathrm{Vgl}$ auch Vasverö Xyk. XXI 48-52. 
die Ansicht, dass re $z^{k}$ 'grün' und dessen hier angeführte Entsprechungen (doch lapp. < fi.) etymologisch wohl desselben 'irsprungs sind, wie syrj. $v e \check{z}$ 'Neid, Begierde' $\sim$ wotj. vož'Zorn, Grimm'.

Wenn also syrj. vez 'grün' mit syrj. vez 'Begierde, Neid' und - wie sich auf Grund der obigen Belege ergänzen lässt - auch 'Zorn, Hass' identisch ist und Ähnliches sich auch auf das Wotjakische bezieht, so entfallen die Bedenken, dass das Adjektivsuffix nicht an ein Adjektiv angefügt sein kann.

Nun müssen wir freilich die Frage nicht mehr in der Form aufwerfen, ob sich denn das syrj. (od. permische) Wort mit der oben festgestellten ursprünglichen Bedeutung 'grün' zur Bezeichnung von 'heilig' eigne, wo wir doch als ursprüngliche Bedeutung von veza (nicht 'grün', sondern) 'verboten, unerlaubt, sündhaft' annehmen mussten. Unsere Belege (beachte besonders vež-kiv uśkedni 'beschreien, einen Schaden anzaubern') zeugen ja dafür, dass das permische Wort gewiss bereits in alten Zeiten (ob eher im Sonderleben der beiden Sprachen, wie Itrosex und Hakulines annehmen; s. MSFOu. XCVIII 194, oder vielleicht noch zur Zeit ihres Zusammenlebens) die neue abstraktere substantivische Bedeutung 'Hass, Zorn' entwickelt hat. Dementsprechend schliesst sich das adjektivische Derivat veža nicht dem Adjektiv vez 'grün', sondern dem $\mathrm{S}$ u b s t a n t i v vez 'Begierde, Neid; Hass, Zorn' an (dieses Substantiv vez ist freilich - wie wir gesehen haben - mit dem Adjektiv vež identisch, hat sich aus diesem entwickelt) und hat die Bedeutung *'zornig, hasssüchtig', also eine Bedeutung, die der Bedeutung 'schädigend, einen Schaden zufügend' gewiss nahe steht, ja sich kaum vom ostjakischen "[etwas tun, was] verboten [ist]", "eine Sünde begehend" und rom samojedischen "sündhaft |sein|" unterscheidet, aus welchen letzteren sich ja wie wir gesehen haben - die Bedeutung "heilig» entwickelt hat.

Syrj. rits'-ko 'Kirche' und vis' 'Opfer'.

1. Das zweite Gried des syrj. Kompositums (Wichm.-Uot.) V S Peč L Le I rit's'-ko, U vit's-ke, P rit's-ku 'Kirche', (Genetz) PO vic-ku 'Kirche (eigentl. heiliges Haus)' ist zweifellos mit jenem Worte identisch, das ebenfalls als zweites Glied in den folgenden syrj.-wotj., bzw. wotj. Zusammensetzungen erscheint: a) s y r j. (Wichm.-Uot.) V S Peč L Le U ker-ka, VU S kier-ka, I ker-ka, P ker-ku 'Haus, Wohnhaus, Stube' (VS Peć U VU I P), 'Stube, Haus mit einem Zimmer' (L Le), (Genetz) PO ker-k-ku. körr-ku 'Haus. Wohnstube' $\sim$ wotj. 
(Munk.) korka 'Haus, besonders mit Ofen versehene Winterwohnung', (Wichm.) korka 'Haus, Stube'; b) w o t j. (Munk.) S vu-ko 'Mühle', (Wichm.) J vuko'Wassermühle, Mühle' (s. z.B. Wicim. WotjChrest. 77, Uotila Kons. 14, 200, SyrjChrest. 98). Das Wort -ka, -ko usw. hat die Bedeutung 'Haus'; syrj. ker-ka usw. bedeutet eigentlich 'Balkenhaus', wotj. vu-ko 'Wasserhaus'.

Was nun das erste Glied in syrj. vi t's'ko betrifft, so kann dieses auf ursprünglicheres *vid'z- zurückgehen, dessen auslautende Affrikata vor dem stimmlosen Anlaut (dem $k$-) des zweiten Gliedes gleichfalls stimmlos geworden ist. Dieses *vidźwird gewöhnlich mit syrj. (Wichm.-Uot.) V S L U P vìdź, I vid'z'die Fasten', (Genetz) PO $v i i_{\tilde{j}}^{\prime}$ 'Fasten' identifiziert (s. GENETZ Ostpermische Sprachstudien 30, Uot. Kons. 400, 408, Wichm.-Uot. 333, Torvones FUF XXXII 22).

2. Diese Benennung vit's-ko 'Kirche' ist schon in den syrj. Sprachdenkmälern belegt und geht wohl auf die aus dem XIV. Jh. stammende Übersetzung des Bekehrers der Syrjänen zum Christentum, des Bischofs Stephan, zurück. Das Wort ist dort nach G. S. LYTKIx (ZyrjKr. I 35) vitśko, nach V. I. LYTKIN (Drperm. 68, 73, 126) vidźko zu lesen.

Die Deutung dieser alten Benennung als "Fastenhaus" mutet gewiss befremdend an. GENETz (a.a.O.) beruft sich auf karel. pühä, das sowohl 'heilig', als auch 'Fasten' bedeutet, und will wohl die Bedeutungsentwicklung 'Fastenhaus' - 'heiiiges Haus' durch die Parallele 'Fasttag' 'heiliger Tag, Feiertag' erklärlich machen. In den permischen Sprachen deutet jedoch nichts auf die Möglichkeit einer derartigen Bedeutungsentwicklung; 'heilig' heisst im Syrjänischen bereits in den Sprachdenkmälern - wie wir eben dargelegt haben - veža (von einem Zusammenhang dieses Wortes mit vid'z kann keine Rede sein), und im Wotjakischen ist in dieser Bedeutung (Munk.) biddzim usw. 'erhaben, herrlich. majestätisch, durchlauchtigst, hehr; heilig, allerheiligst'. sowie (Munk.) izge usw. 'heilig, fromm' gebräuchlich. Für das erstere wotj. Wort, dessen Grundbedeutung wohl 'gross' ist, vgl. Uot. Kons. 230-1; das andere Wort, izge, ist - wie Muskícsi VotjSz. 50 bemerkt - tatarischen Ursprungs (vgl. z.B. kaz. izge 'heilig, fromm, gut' usw. ${ }^{1}$ ).

3. Noch mehr verliert die Annahme einer Bedeutungsentwicklung 'Fasten' $\rightarrow$ 'heilig' an Wahrseheinlichkeit, wenn wir das System der christlichen Terminologie im Syrjänischen in Betracht ziehen. Bischof Stephan hat - wie Uotis FUF

1 Dieses Wort, oder richtiger: ein in diese Wortfamilie gehörendes Wort ist auch die Quelle des ung. ëgy in ëgyház. 'Kirche' (eig. 'heiliges Haus'); s. Gonbocz BTLL. 135, Németh KCsA I 242-3, Ligeti MNy. XXIX 278-9. 
XXVI 188 ausführt - »einige Wörter zur christlichen Terminologie geschaffen, bzw. schon vorhandene Wörter in einer neuen christlichen Bedeutung gebraucht». Eine dritte Quelle bilden dem Russischen entlehnte Benennungen oder diese widerspiegelnde Lehnübersetzungen. Nun gab es bei den Syrjänen der heidnischen Zeit gewiss keine heilige Stätte, die ihren Namen vom Fasten bekommen hätte, auf das Russische geht ja eine derartige Benennung auch nicht zurück, oder sollte Bischof Stephan gerade diesen syrj. Namen gewählt und hierbei nicht daran gedacht haben, dass ein Verknüpfen des Namens der Kirche mit den Fasten keine grosse Anziehungskraft besitzen konnte und wohl nicht sehr zum Kirchenbesuch aneifern würde?

Da Uotila und Torvonex gewiss auch hieran denken mussten, dürfen wir wohl annehmen, dass sie das Wort vit's-ko nicht unmittelbar mit vid'́ 'Fasten', sondern eigentlich mit dem Nomen-Verbum-Stamm ridź verbinden wollten, wie ja Uotila SyrjChr. 179 - fünf Jahre nach dem Erscheinen des Kons. - vitśko unter dem Stichwort vidźni 'schützen, bewahren' anführt (wo er freilich auch vid'ź 'Fasten' einreiht). Syrj. ridź-als Verbum hat ja Bedeutungen, die es nicht Iıur der Bedeutung 'fasten', sondern auch anderen Bedeutungen nahe bringen, die sich besser mit der Bedeutung von vit's-ko vereinbaren lassen. WiEjemans führt als Bedeutungen von syrj. vidźny die folgenden an: 'sehen, besehen, betrachten, übersehen, U̇bersicht haben, - halten, beobachten, nicht übertreten, - hüten, behüten, beaufsichtigen, bewahren, bewachen, erhalten, retten, befreien, - halten (Tiere)' und als Bedeutungen eines anderen vid́ng verbrauchen, ausgeben, verleben, verausgaben, gebrauchen, anwenden'. Lytkis (Drperm. 125) trennt vidź 'держи' von ivd'ź- 'schauen'. Bei Wichm.-Uot. finden wir folgende Angaben: V S Peč L I P vidźni, U vid'źnis 'держать, halten' (V S L P U), 'хранить, bewahren, hüten, beschützen' (V S U), 'varoa, bewahren' (I), 'hüten (z.B. Kühe, Pferde)' (Peč), 'nachsehen, pflegen (z.B. ein Kind)' (S); 'sehen, schauen, besichtigen' (L), 'fasten' (I); '(Geld) verwenden, verschwenden' (L P). Vgl. noch (Wied.) vidźalan, vidźalyś 'enthaltsam, mässig'. Wenn wir num bedenken, dass das entsprechende wotj. Wort (Wichm.) G vozîn n̂., M voźni, J MU vozini 'halten; beschützen', (Munk.) S vo ž-, M voź-, G J K voz'- 'halten (etw. irgendwo); erhalten, bewahren, schützen, verteidigen; beobachten (eine Zeremonie); umgehen, verfahren (mit etw.), behandeln (jmden)' debeutet und $\mathrm{S}$ kis vo ̌n $i$ die Bedeutung 'fasten' hat [eig. 'Trockenes halten'; vgl. syrj. V S I U kos-lun 'Fasttag' [eig. 'trockener, magerer 'Tag'], (U auch:) 'die Fasten'], so kann dies freilich auf ein Verknüpfen ron 
vit's-ko mit vid’źni deuten (hierüber s.w.u.) und sogar - falls vid’́ 'Fasten' und vidźni tatsächlich einen gemeinsamen Stamm darstellen - auch das Verbinden von vit's-ko mit vid'z' 'Fasten' erklärlich machen. Es muss aber Bedenken erwecken, dass im Wotjakischen neben voźni (das dem syrj. vid'zni regelrecht entspricht) das Wort für 'Fasten' mit $i$ lautet: (Munk.) S viź, (in einer Quelle: vidź) (viź kutini od.viźani 'Fasten halten, fasten'), (Wied.) viź, vidź (= syrj. vidź) 'Fasten', badzim v. 'die Fasten vor Ostern', rožtovu-v. 'die Fasten vor Weihnacht', viźan 'Fasten', viźany (= syrj. vidźalny) 'fasten'. Das wotj.-russ. Wörterbuch von 1948 hat viź 'пост', viź vozini 'поститься' (das russ.-wotj. Wbuch von 1956 hat kös voźon, post 'пост', kös (od. post) voźini 'поститься', eig. 'Fasten (Trockenes) halten'). Dies würde darauf deuten, dass syrj. vid'z' 'Fasten' von vidźni 'halten, bewahren, schützen' zu trennen ist; in diesem Falle kann also vit's'ko - wie wir oben ausgeführt haben - auch mittelbar nicht von vidź 'Fasten' hergeleitet werden. Wir müssen aber noch w.u. auf diese Zusammenstellungen zurückkehren.

4. Es gibt freilich auch andere Deutungsversuche für syrj. vit's-ko. Da mehrere Sprachen (unter ihnen auch finnischugrische) - einer natürlichen Anschauungsweise, bzw. Denkart entsprechend - die Kirche "heiliges Haus" oder "Gottes-

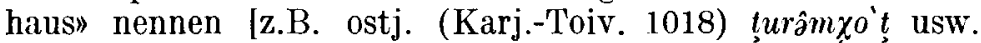
"Kirche, Gotteshaus", wog. (Romb.) я.пиннгкол 'церковь' usw.; auch das russ. церковь, deutsch. Kirche bedeuten ursprünglich "Gotteshaus»], suchte man auch syrj. vit's-ko auf eine ähnliche Weise zu deuten.

So hat bereits Budenz (Nyr. I 73 und MUSz. 808) das erste Glied von vit's-ko mit einem wotj. Worte zusammengestellt. das 'Opfer; Gebet' bedeutet. Dieses Wort erscheint bei Muskícsi in der Form $\mathrm{S}$ voś, $\mathrm{K}$ viüś 1. 'Opfer'; 2. 'Gebet'; 3. 'Religion, Gottesdienst, Religionsübung' (hiervon: S vos'-kill, K vüis'kel 'Gebet', S. voss-korka 'Gebethaus, Tempel'; S vəśaśkon, [K] vüsaśkon 'Gebet; das Beten, das Opfern'), bei Wichmans (WotjChrest. 127) in der Form G U veśs, MU vös' 'Opfer, Gebet', hiervon veśànî usw. 'opfern, beten'; G vośaśkịnị, J vöśaśkini 'beten', J vöśáśkon-kil, J MU vöśáśkon-kivu 'Gebet'. Diese Zusammenstellung, die ja semasiologisch völlig befriedigend ist, billigt auch V. I. LyTkIN (Drperm. 73, 126). Die ursprüngliche Bedeutung von vit's-ko wäre demnach - wie Lytkin bemerkt 'молитвенный дом', bzw. 'дом для жертвопрнношения', d.h. 'Bethaus', bzw. 'Haus zum Opferdarbringen'. Auf diese Weise wäre also auch hier ein alter heidnischer Termin zur Anwendung gelangt.

5. In Verfolgung dieses Gedankens (*vidź[-ko] wotj. vöś) 
glaubte aber Lytkis, seine Deutung noch durch das Heranziehen eines anderen alten syrj. Wortes stützen zu können, indem er (a.a.0.) auf das in den Sprachdenkmälern vorkommende altsyrj. vis 'жертва; Opfer' hinwies. G. S. LYTKIN zitiert nicht den Lepechinschen Text, der demjenigen Teil der Jevgenijschen Texte entspricht, in dem dieses Wort vorkommt, so dass wir dieses Wort bei ihm umsonst suchen; das Wort konnte bis nun auch aus den syrj. Mundarten nicht nachgewiesen werden. Nichtsdestoweniger ist das Wort und auch seine Bedeutung einwandfrei bezeugt. In der Photokopie der Jevgenijschen Texte (s. Drperm. 165) sind nicht nur die syrj.

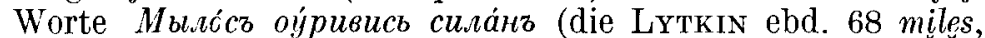
uri, viś, śilan liest) deutlich sichtbar, sondern es ist dort auch der der Übersetzung zugrunde liegende Originaltext: "Ми́лость ми́ръ же́ртва и́п'́ніе» eingeflochten. V. I. LYткIN identifiziert nun nicht bloss das wotj. veś, vös 'Opfer, Gebet', sondern auch dieses altsyrj. vis' 'Opfer' mit dem ersten Glied von vit's-ko, somit auch altsyrj. viś mit wotj. veś, vöś.

So ansprechend diese Zusammenstellung anscheinend ist und Lytkis sucht sie auch lautlich zu begründen - bestehen dennoch gerade in lautlicher Beziehung gewisse Schwierigkeiten.

Der Vokalismus würde stimmen: die Lautvertretung wäre dieselbe wie z.B. in syrj. $k i$ : V S Peč L U IU $i z-k i$, I $i z-k i, i z-k i$, IU is-li 'Mühlstein' $\sim$ wotj. (Wichm.) U M G ke, MU J kö : vuko-kï id., (Munk.) S k9, K kü 'Mühlstein' (s. Uot. Kons. 252); vgl. auch teilweise syrj. (Wichm.-Uot.) V S L U P vidź, I vid'ź 'Wiese' wotj. (Wichm.) G MU U voz, J M S vodz' 'Wiese, Heuwiese', (Munk.) S vo ̌̆, M vo $\tilde{3}, \mathrm{~K}$ voź 'Heuwiese, Mähwiese' (s. auch ToIvonen Affr. 162, ITKonen FUF XXXI 182, 311).

Bezüglich des Konsonantismus beruft sich LyTkIs (op.c. 73) auf Fälle, wie syrj. tcliśs wotj. toledtź, toleź 'Mond, Monat'; syrj. I pelidź, V VU S L Le Peč peliś 'Vogelbeere' $\sim$ wotj. M paled ź, U J G paleź, MU J påuéz, MU påuez, U paleź usw. id.; syrj. I ameś, U ameś, V amiś, VO (Ustnem) amet's, IU ameś 'Pflugschar, Pflugmesser' wotj. (Munk.) S ame ̌̌, (Wichm.) J amed'́, U MU J M ameź, G omeź, U ameś id. (über diese s. auch Uот. Kons. 170), ferner auf syrj. vid'z' die Fasten' $\sim$ wotj. viź, vidź 'das Fasten', syrj. mi t'śa 'schön' miśtem 'unschön'.

Die angeführten Angaben scheinen tatsächlich den hier in Rede stehenden Lautwechsel $\dot{s} \sim d \dot{z}^{\prime} \sim t^{\prime} \dot{s}^{\prime}$ zu bezeugen und somit die Richtigkeit der Lytkinschen Zusammenstellung zu beweisen. ${ }^{1}$ Es stünde auch der Annahme nichts im Wege, dass

${ }^{1}$ Syrj. $m i_{\text {( }} t^{\prime} s a \sim$ miśtem ist jedoch gewiss anders zu erklären. Vgl. z.B. Le veśni, Pr vessni 'machen' zum Inf. anderer Mundarten: oetśni 
eben in vitś-ko neben viś die Variante mit $t s ́ d z$ (vgl. UoT. Kons. 170, 171, 159) erhalten geblieben sein kann. Dennoch darf ein wichtiger Umstand nicht ausser acht gelassen werden. In den oben angeführten Fällen erscheint im Wotjakischen immer auch $t^{\prime} s\left(t^{\prime} s\right)$, bzw. $d \dot{z}, \dot{z}$. Bei ursprünglichem * $t^{\prime} s$ tritt im Wortinnern nur in $\mathrm{C}$ und $\mathrm{K}$ (in einem Worte: nemis' 'Deutscher' auch in MU) $s$ auf, doch auch hier nicht ausschliesslich (s. Uot. Kons. 153-4; vgl. auch ebd. 305-7, 310), bei ursprünglichem ${ }^{*} d z$ tritt $s$ ebenfalls nur in $\mathrm{U}$ und $\mathrm{K}$ auf, doch auch hier neben $\dot{z}$, in $\mathrm{K}$ auch $d \dot{z}$ (s. Uot. Kons. 157, 170-1; vgl. auch Torv. Affr. 229-30), abgesehen ron den vereinzelten Fällen, wo das $\dot{s}$ (statt $z$ ) durch satzphonetische Verhältnisse (vor einem stimmlosen Konsonanten) bedingt ist. (S. auch Wichim. WotjChrest. ${ }^{2}$ 140, 162; JEMEL'JAxov GrVotj. 125, zu der hier zitierten Form kižzoś vgl. aber Musk. VotjSz. 68,151 .)

Einer Annahme, dass in wotj. ress usw. überall die schwache Stufe der ursprünglichen Affrikata vertreten sei (hierzu vgl. z.B. Torv. Affr. 256, Uot. Kons. 171), widersprechen die oben erörterten Lautverhältnisse.

Im wotj. Worte für 'Opfer, Gebet' dürfte sich also, wenn es tatsächlich mit syrj. vit'ś-ko od. *vidź-ko zusammengehören würde, eine Form mit $s$ - wenn überhaupt - so höchstens in $\mathrm{K}$ und $\mathrm{U}$ vorfinden. Demgegenüber erscheint aber in diesem wotj. Worte ausnahmslos - auch in $\mathrm{S} \mathrm{G} \mathrm{J}-\dot{s}$, und nirgends $\dot{z}$ oder eine Affrikata. Für das wotj. Wort muss also ursprüngliches * ${ }^{\prime}$ angesetzt werden; es kann demnach - u.zw. mit Recht - mit syrj. vis 'Opfer' zusammengestellt werden, doch von syrj. vit's-kio muss das wotj. veś und folglich auch das mit diesem zusammenhängende altsyrj. viś getrennt werden.

6. Der Vollständigkeit wegen sei hier noch auf zwei Formen hingewiesen, denen aber bezüglich der Etymologie unseres Wortes gewiss keine Bedeutung beigemessen werden muss. ('ASTRÉx hat in I neben vidzj (lies: $v i d z ')$ 'pratum' vic'ko 'templum' (so auch auf S. 119), welche Form sowohl ritślio als auch vițško gelesen werden könnte (vgl. Castréx p. VIII, 3 und

od. Präs. Sg. 1. P. auch Le Pr vectsa; s. auch Uot. Kons. 305-7, 310, Castré n Gramm. 3-4. - Wir wollen aber gleich bemerken, dass bei syrj. viś 'Opfer' nicht mit der Möglichkeit einer ähnlichen Vereinfachung einer Konsonantengruppe (*-t's + Konsonant) gerechnet werden kann. Das in der oben zitierten Wendung auf sis folgende Wort (silan) lautet mit $s$ an; hier lag also kein Grund vor, *vitś $+\dot{s}$ zu viśss- zu vereinfachen. Im Gegenteil: eine derartige Lauterscheinung wäre eher in der Form vit's-ko > * viś-ko erklärlich gewesen; vgl. z.B. Le $k \prime s$-ke 'irgendwie'

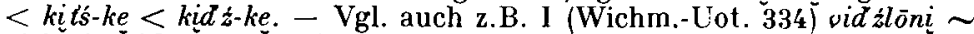
viźlöni $\sim$ Vm (eig. Aufzeichn.) vi żlōni, usw. 
z.B. köc' 'lepus' usw.), und Rogov führt in beiden Teilen seines permjak. Wörterbuches für 'Kirche' zwei parallele Formen an: vitskku und vittskik.

Schliesslich erinnern wir nochmals daran, dass - wenn vit's-ko das Wort vis' enthalten würde - hier in erster Reinn *vis-ko zu erwarten wäre, und nicht vit's-ko die allgemein verbreitete Form wäre (s. oben S. 174).

7. Es waren vielleicht eben die angeführten Schwierigkeiten und Erwägungen, die auch Uotila (SyrjChrest. 179 und Wichm.-Uot. 333, 336) bewogen haben, syrj. vis' von vit's-ko zu trennen. Freilich müssen wir aber Uotilas Deutungsversuch, dieses syrj. viś 'Opfer' sei mit syrj. viś (z.B. viś setni 'Nachricht od. Kunde geben') identisch, ablehnen. Das letztere Wort (vgl. auch das davon abgeleitete syrj. vistalni 'sagen, erzählen') ist gewiss ein Lehnwort und geht auf russ. весть 'Nachricht' zurück (vgl. Kalima RLS 166, G. S. LytkiN ZyrjKr. II 49, Popov-Lytkin 33; s. auch V. I. Lytkin Uč. zapiski Rjaz. Pedag. Inst. N:o 10, 1955, p. 120, obzwar er Drperm. 126 auch die Uotilasche Deutung für möglich hält). Zum Schwund des $-t$ in der auslautenden Konsonantenverbindung vgl. KAL. RLS 13, MSFOu. LXVII 155; vgl. auch das oben zitierte altsyrj. miles < russ. милость (über dieses jedoch anders LYTk. Drperm. 117, 137).

8. Es muss noch ein Versuch zur Erklärung von vit's-ko erwähnt werden. BuDEnz wollte das erste Glied dieses Wortes zuerst (Nyr. I 73) mit ung. ëgy in ëgyház 'Kirche' (eig. 'heiliges Haus') verbinden, doch im Syrj. erscheint in allen Dialekten anlautendes $v$ (vor $i$ ), dem im Ung. nicht vokalischer Anlaut entsprechen könnte (s. z.B. ToIv. FUF XXIX 160), und ausserdem ist ja das ung. Wort seitdem befriedigend als türk. Lehnwort erklärt worden (s. oben S. 171 Fussnote; vgl. auch BALÁzS ALH VI 145).

9. Die angeführten Deutungen können uns also nicht befriedigen. Wir können aber auf einen Umstand aufmerksam machen, der gewiss schwer ins Gewicht fällt und u.E. zum Klären unseres Problems beitragen kann.

Die altsyrj. Sprachdenkmäler kennen ein Wort viddzan (in den Lepechin-Jevgenijschen Texten: виза́нъ) in der Bedeutung 'спасение; E r lös u n g; H e il' (s. Lytк. Drperm. 67, 126, 165). (Das zweite vi dzzana ebd. 69, 167 ist nicht klar.) Das Wort ist natürlich eine Ableitung von vidźni, das - wie wir oben gesehen haben - 'halten, bewahren, schützen', aber auch heute noch auch 'retten, befreien' bedeutet. Bischof Stephan hat aber nicht nur das Derivat vid dźan in die kirchliche Terminologie aufgenommen; das oben (S. 172) erwähnte vidź (Lep.- 
Jevg. ви́зъ; s. Drperm. 68, 73, 125, 165), das er - in richtiger Kenntnis der hebräischen Etymologie des entsprechenden russischen осанна im kirchenslaw. Originaltext - im Sinne von 'schütze [uns]!' (eig. 'hilf doch!') anwendet, beweist ebenfalls, dass er dieses Wort zur Wiedergabe von 'retten, erlösen', bzw. 'Erlösung, Heil' geeignet fand. Der Gedanke liegt wohl nahe, dass Bischof Stephan diesen Ausdruck wählte, wenn er die Kirche "H a u s de s $\mathrm{H}$ e il s" (im Sinne von "Haus des Heilands", "Haus des Erlösers"; s.w.u.) benennen wollte. Die Bedeutung 'Heil' im religiösen Sinne: 'Seligkeit, Heil der Seele' hat wohl Stephan diesem Worte (nach russ. Muster) gegeben.

Bevor wir noch an die Erklärung von Lautgestalt und Bedeutung dieses Wortes *vid' $z$ *'Heil' herangehen, seien noch die hier erwähnten Ausdrücke aus neueren Quellen nachgewiesen: G. S. Lytkin hat im Anhang von ZyrjKr. (im russ.-wotj.-syrj. Wörterverzeichnis p. 34) als Entsprechung von спасти 'retten, erretten' syrj. vidźni..., wotj. vozini..., als Entsprechung von спаситель 'Heiland, Erlöser' syrj. vi džiś, wotj. voźiś; dem russ. спасение 'Erlösung, Heil' entspricht bei ihm svrj. viddzem. Popor-Lytkin hat unter спасать, спасти: vidzni ...,

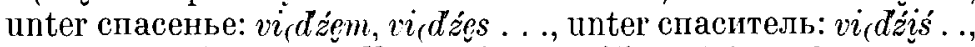
unter спасибо (спаси, Вогъ): jenme, vid'ź . . .! Auch Savvaiтov

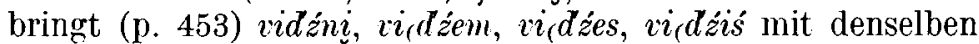
Bedeutungen wie Popov-I ytkin.

10. Wie ist nun das also gewiss mit vidźni zusammenhän-

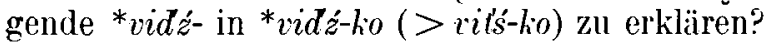

Der Gedanke, dass wir hier einer Neuerung seitens Bischofs Stephan gegenüberstehen, der nach Analogie von russ. спасать, спаситель ('erlösen'; 'Erlöser'): cпас ('Erlöser, Heiland; Christus') aus vidźni 'erlösen' ein Substantiv *vidz' *'Heiland, Erlöser' erschloss, lässt sich vielleicht nicht a limine abweisen. Es ist aber auch eine andere Erklärung möglich, die auch in sprachlichen Tatsachen ihre Stütze findet.

Im I. Bande von VNGy. (Einleitung S. CCLXV) äussert Munkícsi die Meinung, "syrj. vič-ko 'heiliges Haus, Tempel'" enthalte als erstes Glied der Zusammensetzung das Wort

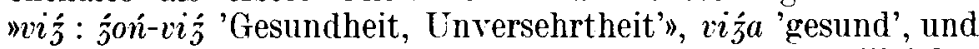
beruft sich, was das Verhältnis von 'gesund' und 'heilig' betrifft, auf das deutsche heilig und heil ('gesund, unversehrt').

Dieser Hinweis auf ein Substantiv vid'z (das also nichts 'Fasten' bedeutet) verdient gewiss ernste Beachtung, und wir wollen dieses Wort näher prüfen.

Das Wichmann-Uotilasche Wörterbuch kennt folgende Angaben (p. 38, 334): vi d'za: V d'ońn-v. ov, I dźon- $v$. ó 'lebe gesund! lebe wohl!' U dzoń-r. ovnis 'gesund leben'. U dzoń- 
vi dzán kot ttśi 'lebe gesund! lebe wohl!' U mun dźon-viddźaen 'geh gesund!' IV S L I U P dźon' 'ganz, heil, unversehrt, unverletzt, gesund' (V S I U P), 'schön, gut (besonders v.d. Wejbertracht)' (L).] WotjChrest. 128 führt WICHMaNN noch syrjI vidź : dźon [o:dzón] vidz' 'Gesundheit' an. - Ich habe folgendes aufgezeichnet: V dźoń-vidz' 'Gesundheit, Wohlbefinden'.

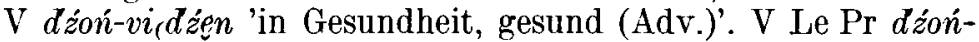

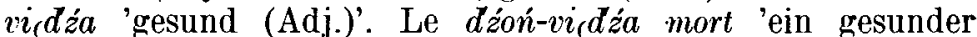

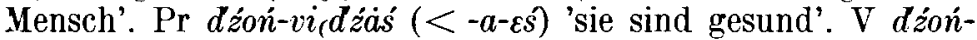

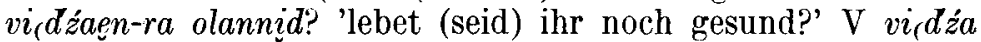
olan! 'sei gegrüsst!' V vi d'źa ola·n-vila·n id. V t'solom-vi,dźa!

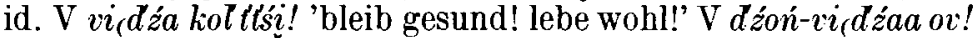
'leb gesund! lebe wohl!' V d'zon-vi,dźaa-na olannid? 'lebet ihr gesund?' - U viź : bur viź-vilin 'in guter Stimmung, in guter Verfassung'. (Zum letzteren Worte vgl. aber auch wotj. viź 'Verstand, Klugheit'?)

Das Hauptwort dźon- $\imath i d z '$ 'Gesundheit, Wohlbefinden, Unversehrtheit; здорове' kennen Savvaitov, Wiedemaxs, PopovLytкin, Wiснм. und Verf. ${ }^{1}$; das Adjektiv d'zon-vi,dźa 'gesund, wohl befindend, glücklich; здоровый' kennen Savvaiтov, Wiedemann, Popov-Lytkin, Sachov, Wichmann und Verf.; (bei dźon-vi, dźa als Adverb: (bei Lytkin:) dźon-vic dźa ol 'живи здорово', dźon-vi dźa olan-vilan 'здорово живешь-можешь', Popov-Lytkin:) dźoń-vi dźa 'здорово, по здорову (в цилости, coxранно)', d'źoń-vi,d'źa olan-tilan 'здорово живешь-можешь' (s. auch WIr'Hм. und Verf.) (diese Formen können aber auch als Adjektive aufgefasst werden). Wiedemaxs und Verf. haben auch dźon-ri,dźa ol (ov)! leb wohl! (Formen, in denen das Adverbsuffix dem Adjektiv vi $i_{d} z ́ a$ angefügt ist). Das einfache $v i_{\text {,d }} a$ a finden wir bei WIEDEMANN (vidźa 'gesund', vidźa olan 'bist du gesund? (Grussformel)', vidźa lokny 'willkommen sein'), bei S̆ACHOV (vid dza olan '(приветствие)'), sowie im Koмирусский словарь von 1948 ( $v i_{\text {d }} d z a$ 'здорово, привет (приветствие)', vidźa olannid 'здравствуйте', vi,dźa tijanli 'привет вам', ri,dźaаśni 'поздороваться, здороваться, приветствовать').

Dieses Wort *vidz, das - wie wir sehen - heute nur in der zitierten Zusammensetzung und in Ableitungen gebräuchlich ist, ist gewiss sehr alt und war auch sehr verbreitet. Schon LEPECHIN hat (1780) визя [lies wohl: viddza; er schreibt auch визедъ, d.h. VU vi,d'zed 'schau', дзеля und зеля, d.h. d'zela 'klein'], das er 'здоров' übersetzt; das Wort hat auch im Wotjakischen seine Entsprechung und ist aus dem Syrjänischen ins Ostjakische und mit dessen Vermittlung ins Wogulische und

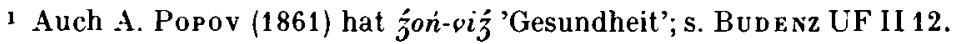


Samojedische eingedrungen. Im Wotjakischen entspricht ihm nämlich - wie schon Muxkícsi ÁKE 364 hierauf aufmerksam gemacht hat -: voźo: $\mathrm{K}$ voźo karene, deč-voźo karene, $\mathrm{G}$ voźoburo karini 'jemanden begrüssen, bewillkommen' (s. auch VotjSz.). Das wotj. Wort hatte aber bereits G. S. LYTKIN mit dem syrj. verglichen; in seinem Wörterbuch (p. 48) weist er unter syrj. vi,dźa auch auf wotj. voź 'миръ', voźo-bu rokarini 'привътствовать' hin und unter wotj. voź (ebd. p. 121) führt er für dieses wotj. Wort die Bedeutungen 'миръ, безопасность' an. Auch Wiedemaxs hat wotj. voź 'Friede, Sicherheit', ferner' voźo karyny, voźo buro karyny 'grüssen, bewillkommnen'. Betreffs der ostj. Formen (Ni ự ż $\dot{a}^{\prime}$ 'heil!', uiqza ut $\dot{a}^{c}$ 'heil!, lebe gesund!', wǔsa 'Gruss beim Begegnen' usw.) s. AHLqv. No 117, 152, Steinitz OstjGr. 167, Tolv. FUF XXXII 22; bezüglich der auf diese zurückgehenden wog. (paśä $<\mathrm{NO}$ $p a-u s a)$ und juraksamojedischen $(\beta i s, s a p)$ Formen $s$. noch Musk. VNGy. II 0680, LeHT. Wb. 74.

Die Mehrzahl der obigen Belege (und auch das Wotjakische) sprechen dafür, dass wir es hier mit einem Substantiv ridź zu tun haben (dźoń vidź hätte also ursprünglich 'vollkommene 'resundheit' bedeutet, und d'źon-vi,dźa hätte die Bedeutung 'vollkommene Gesundheit habend', d.h. 'vollkommen gesund' als Adjektiv); einige Belege jedoch können auch in dem Sinne gedeutet werden, dass vi ${ }^{\prime} z^{\prime} a$ ein Adverb aus einem Adjektiv * ridź darstellt. Nomina sind ja in den fiugr. Sprachen nicht selten sowohl als Substantive als auch als Adjektive gebräuchlich. So bedeutet z.B. syrj. šog 'Trauer, Sorge, Betrübnis ...', aber auch (sogar in ein und derselben Mundart) 'traurig, betrübt; müde', und dementsprechend kann šoga einerseits ein vom Substantiv šog abgeleitetes Adjektiv ('traurig; maestus'), andererseits das Adverb zum Adjektiv šog sein ('maeste'; z.B. VU šoga pondas ovni 'sie beginnt bekümmert zu leben'). (Auch das entsprechende wotj. sug kann nach VotjSz. 497-8 beide Funktionen versehen. Vgl. auch oben vež 'grün' und rez 'Berierde, Neid; Hass, Zorn' und w.u. dźet's.) Jedenfalls wurde wenn auch vidźz ursprünglich auch ein Adjektiv gewesen sein sollte - mit der Zeit die Form vi,dza so allgemein, dass sie vielleicht alleinherrschend wurde und als erstarrte Formel (s. den aus dem Komi-russ. Wbuch zitierten Beleg) auch ins ostjakische (und von hier auch in andere Sprachen) einzudringen vermochte.

Auf jeden Fall können wir feststellen, dass die in den zitierten Grussformeln auftretenden Ausdrücke syrj. dźoń- $v i$,dźa, wotj. deč-voźo gewiss aus Bestandteilen mit synonymen Bedeutungen zusammengesetzt sind. Heute lautet die wotj. Begrüssungsformel (der - wie es scheint - die ältere Form weichen musste): 
d'źet'śbur liarini 'ириветствовать, здороваться', dzet'śburaśkini 'здороваться с кем' (s. das russ.-wotj. Wörterbuch aus dem J. 1956; s. auch Munk. VotjSz. unter ž ě́-bur). Da (Wichm. WotjChrest. 54) G dzéet's, M J MU dźét's, J (auch:) dźés' 'gut, glücklich, schön; Glüick' bedeutet, ist der Sinn des wotj. Grusses 'friedlich (od. sicher, od. ursprünglich vielleicht: *gesund) [und] gut [od. glücklich]!' (s. auch ÁKE 364) und der des syrj. Grusses - wie wir oben gesehen haben - 'heil und gesund!'. Eine ähnliche Grussformel zitiert Munkácsi (a.a.O.) aus dem Tawda-Wogulischen: šaw-pisèn alánten-li?' 'bist du gesund, heil (живой, здоровой живешь-ли)?'

Aus dieser Bedeutung 'gesund' (bzw. 'Gesundheit') leitet nun Muxkácsi die Bedeutung 'heilig' (bzw. 'Heil') ab und beruft sich auf eine Bedeutungsentwicklung, die - der oben unter veža erwähnten ähnlich - aus mehreren Beispielen bekannt ist. So ist die Grundbedeutung des deutschen Wortes IIeil das 'Freisein von körperlicher Verletzung' (vgl. heilen 'gesund machen'), und heilig bedeutet eig. 'voll guter Kraft', wobei das Wort seine heutige Bedeutung ('sanctus') durch die Stufe 'gut, glücklich' bekommen hat (s. KLUGE-GöTz ${ }^{11}$ ). Auch das wog. jelpin 'heilig' hat ursprünglich 'heil, vollkommen, fehlerfrei' bedeutet (s. Munk. VNGy. I CCLXV; s. auch BUDExz MUSz 807), dem russ. святый 'heilig' entspricht ai. ved. çvāntás 'gedeihend' (VAsmer 597). S. auch ÁKE 364. (Ins Südlapp. wurde sowohl das 'heilig', als auch das 'heil' bedeutende skand. Wort übernommen: LAGERcr. LappWb. 26 ajleks 'heilig' und ăjliєs 'heil, gesund'.)

Nach (od. richtiger: auf Grund von) Munkácsis Erklärung bedeutet also syrj. vit'ś-ko 'Haus des Heils, Haus der Heiligkeit' (?' 'heiliges Haus').

11. Wir sind oben zum Resultat gelangt, dass laut Zeugnis des aus vidźni erschlossenen ${ }^{*} v i d z$ das syrj. Wort für Kirche ursprünglich 'Haus des Erlösers' od. 'Haus des Heilands' bedeuten konnte. Doch nichts steht ja der Deutung im Wege, dass das erschlossene *vidlź nicht 'Erlöser, Heiland', sondern 'Erlösung, Heil' bedeutet hat, wie wir auch oben bemerkt haben.

Die zwei Bäche münden in einander und setzen nun - einer den anderen verstärkend - ihren Lauf gemeinsam fort. Wir haben oben ein syrj. Wort ${ }^{*}$ vid'z supponiert und nun sehen wir, dass dieses *vid'z in Wirklichkeit vorhanden ist, denn [dzoń-] vid'ź ist ja eben derselbe (Nomen-Verbum-)Stamm, den wir in vidźni sehen. Und dieses vidź 'Heil' ist ein uraltes Wort, es hat seine Entsprechung im wotjakischen voz' 'Friede, Sicherheit'. Dass vidz', das zuerst 'Gesundheit, Heil' bedeutet hat, 
in der Liturgie im Sinne von 'Erlösung, Heil' angewendet wurde, dazu hat - wie gesagt - ausser dem russ. Muster auch Bischof Stephans bewundernswerter sprachschöpferischer Geist das Seinige beigetragen.

12. Unsere Ausführungen bedürfen noch einiger Ergänzungen.

Das syrj. vidz'- ind vitś-ko hat Budexz (MUSz. 807-8) mit dem zweiten Teil von ung. üd-röz 'sospes, salutatus, henedictus; selig, willkommen' zusammengestellt, ebenso Muxkícsi (ÁKE 363-5) (der auch syrj. vi ̌ 'Fasten' und jweifelnd auch věza 'heilig' hierherrechnet) und andere (z.B. Horgen Magyar szavak története $175-6$; s. Bír(zi SzófSz. 325), Wichmaxs wiederum hat (WotjChrest. 128) syrj. vidź in dzon-cidz' resundheit' mit wotj. G MU U viz' 'Verstand, Klugheit' verbunden. Was nun ung. üd-vöz betrifft, hat BÁRczi (a.a.O.) gewiss recht, wenn er erklärt, die Budenz-sche Deutung sei nicht überzeugend; gegen die Wichmannsche Zusammenstellung sprechen sowohl Bedeutung als auch Form der miteinander verglichenen Wörter, denn der Stamm des wotj. Wortes ist viźm- (z.B. vizmo 'klug'; s. auch VotjSz. 670) und das Wort hat nach Muskícsi auch die Form viz.

Auffallend und unklar ist schliesslich eine Angabe, die WikI)MANN in seinem Wörterbuche (p. 565) unter den Ergänzungren und Berichtigungen anführt: vidź-vištalny 'zeugen', v.vištalöm 'Zeugnis', v.-vištalyś 'Zeuge'. Dieses vidź erklärt sich wohl auf Grund der Bedeutungen von (Wied.) vidźlalny (vidzlavny) 'untersuchen, erfahren, - bezeugen, bestätigen, bekräftigen', (Wichm.) I rid'źlōni 'besehen, betrachten', I viźlōni 'probieren, versuchen, prüfen, untersuchen' (vgl. auch Wied. vidlalny pöveritny 'bezeugen', Popov-Lytkin vidlini, cidlalni 'сви;'Łтетьствовать'), so, dass wohl vidź-viśtalny eig. 'Bezeugrung aussagen, erklären' bedeutet.

Wir waren gezwungen, mehrere Wörter in unsere Untersuchungen einzubeziehen, infolgedessen sind unsere Ausführungen gewiss nicht genügend übersichtlich geworden. Wir wollen deshalb zum Schluss anf die im Titel unseres Beitrages vorgeführten Wörter zurückkommen und zusammenfassend feststellen: syrj. vits'-ko 'Kirche' und syrj. ris' 'Opfer' haben unseres Erachtens nichts miteinander zu tun; vitś-ko enthält als ersten Teil der Zusammensetzung das Wort syrj. vidz 'Gesundheit, Heil' wotj. voz' 'Friede, Sicherheit', während syrj. ris' 'Opfer' mit wot.j. veś, vöś 'Opfer, Gebet' zusammenzehört. 